\title{
Game-Theoretic Analysis of Multibandwidth Channel Selection by Coordinated APs in WLANs*
}

\author{
Kohei HANADA $^{\dagger a)}$, Student Member, Koji YAMAMOTO ${ }^{\dagger}$, Senior Member, Masahiro MORIKURA ${ }^{\dagger}$, Fellow, \\ Koichi ISHIHARA ${ }^{\dagger \dagger}$, and Riichi KUDO ${ }^{\dagger \dagger}$, Members
}

\begin{abstract}
SUMMARY As the demand for high-throughput communications in wireless LANs (WLAN) increases, the need for expanding channel bandwidth also increases. However, the use of wider band channels results in a decrease in the number of available channels because the total available bandwidth for WLAN is limited. Therefore, if multiple access points (APs) are in proximity and the cells overlap, it is difficult for each AP to use an orthogonal channel and competition increases between APs using the same channel. Coordination of APs is one promising approach; however, it is impractical to control all APs in WLAN systems. To cope with this problem, we proposed to analyze throughput performances of a multibandwidth channel selection by the coordinating APs at Nash equilibria, which can be considered as operating points for independent channel selection by APs. To clarify the effect of coordinating APs, we assume a simple scenario where the cells of three or more APs overlap, and each AP can select multibandwidth channels to maximize their own throughput. Through game-theoretic analysis, we find that the coordinated APs are able to select channels more effectively than if each AP independently selects channels. Consequently, the total throughput of the coordinated APs at Nash equilibria is significantly improved.

key words: wireless LAN, multibandwidth, channel selection, IEEE 802.11ac, overlapping BSS, game theory
\end{abstract}

\section{Introduction}

Wireless LANs (WLANs) have become increasingly popular, and one of the recent trends for WLANs involves the offloading of mobile cellular traffic. Thus, multiple access points (APs) are deployed by different operators. Therefore, cells formed by APs often overlap, and such cells are called overlapping basic service sets (OBSS). When the cells of multiple APs overlap and then select the same channel, inter-cell contention occurs. This inter-cell contention causes a decrease in throughput; therefore, in such a scenario, channel selection is important.

In IEEE 802.11 ac [1], [2], 80 MHz and $160 \mathrm{MHz}$ channels will be added to the available channel bandwidth of IEEE 802.11n [3]. That is, IEEE 802.11ac will support mandatory bandwidths of $20 \mathrm{MHz}, 40 \mathrm{MHz}$, and $80 \mathrm{MHz}$ as well as an optional bandwidth of $160 \mathrm{MHz}$. Because the total available bandwidth in a WLAN is not changed, expand-

\footnotetext{
Manuscript received October 11, 2012.

Manuscript revised January 31, 2013.

${ }^{\dagger}$ The authors are with the Graduate School of Informatics, Kyoto University, Kyoto-shi, 606-8501 Japan.

${ }^{\dagger \dagger}$ The authors are with NTT Network Innovation Laboratories, NTT Corporation, Yokosuka-shi, 239-0847 Japan.

*This paper was presented in part at IEEE VTS APWCS 2012 and IEEE ICCS 2012.

a)E-mail: hanada@imc.cce.i.kyoto-u.ac.jp DOI: 10.1587/transcom.E96.B.1277
}

ing the channel bandwidth causes a decrease in the number of orthogonal channels, rendering it difficult for each AP to select an orthogonal channel. This results in an increase in inter-cell contention.

To overcome the inter-cell contention, a lot of resource allocation methods for WLANs have been proposed, i.e., a static channel assignment [4], dynamic channel assignment [5], [6], and joint AP placement and channel assignment [7]-[9]. These channel assignments have various objectives, i.e., the minimization of traffic at the most congested channel [4], [7], the minimization of interference [5], [9], and the maximization of the total throughput [6], [8]. Although these previous studies have various objectives, they assumed only one bandwidth of channels. To the best of our knowledge, there is no research on multibandwidth channel assignment for coordinating APs in WLAN systems. In contrast to these previous researches, we treat multibandwidth channel selection. Note our purpose is to show the advantages of multibandwidth channel selection and coordination of APs, rather than creating a specific algorithm.

Here, we propose a framework for discussing throughput at Nash equilibria, which can be treated as operating points for independent channel selection. In this method, a centralized controller coordinates multiple APs, and these APs jointly select multibandwidth channels so as to maximize their aggregated throughput when there is an uncontrollable AP which selects channel to maximize its throughput. In order to evaluate the effect of coordinating APs, we initially compare the following two simple scenarios. The first scenario is that there are three APs and each AP operates independently. The second scenario is that only two APs are coordinated by the centralized controller, and the remaining AP is uncontrollable. Through comparison of these two scenarios, we find that the total throughput of the coordinated APs increases. We would like to emphasize that the main purpose of this paper is to assess the coordination capability of APs and multibandwidth channel selection, and not to establish detailed channel selection methods. Thus, the information required for channel selection is assumed to be known by all the APs, and detailed information collection and channel selection schemes are beyond the scope of this paper.

To show the effectiveness of the above-mentioned coordinating APs and multibandwidth channel selection, a game-theoretic analysis is conducted. This is because game theory formulates interactions provided by the multiple 
decision-making entities. Game theory is a promising tool for wireless communications because there are many kinds of interactions in wireless networks such as contention and interference [10].

This paper is organized as follows. Section 2 presents an overview of IEEE 802.11 ac and game theory. In Sect. 3, a system model is introduced, and in Sect. 4, a game-theoretic formulation of assumed channel selection is conducted. Details of the numerical value of the utility function are discussed in Sect. 5. In Sect. 6, we discuss the effectiveness of multibandwidth channel selection by coordinating APs through numerical evaluations. Finally, we present our conclusions in Sect. 7.

\section{Background}

\subsection{IEEE 802.11ac}

To increase throughput, in IEEE $802.11 \mathrm{ac}, 80 \mathrm{MHz}$ and $160 \mathrm{MHz}$ bandwidth channels will be added to the available channels, and the maximum number of spatial streams will be increased to eight [1], [2]. Using these maximum values, we will be able to achieve a data rate per channel of over $1 \mathrm{Gbit} / \mathrm{s}$.

In IEEE $802.11 \mathrm{ac}$, the channel arrangement of each channel bandwidth in Japan will be regulated, as shown in Fig. 1. Note that an AP can use a $40 \mathrm{MHz}$ channel from $5.17 \mathrm{GHz}$ to $5.21 \mathrm{GHz}$ but cannot use a frequency from $5.19 \mathrm{GHz}$ to $5.23 \mathrm{GHz}$ as a $40 \mathrm{MHz}$ channel. In IEEE $802.11 \mathrm{ac}$, each AP should reserve one $20 \mathrm{MHz}$ channel in the used bandwidth as a primary channel, regardless of the selected channel bandwidth. For example, when an AP sets the third $20 \mathrm{MHz}$ channel in Fig. 2 as the primary channel, the fourth $20 \mathrm{MHz}$ channel should be set as the secondary channel, and the primary and secondary channels are aggregated to a $40 \mathrm{MHz}$ channel to use. Similarly, the first and

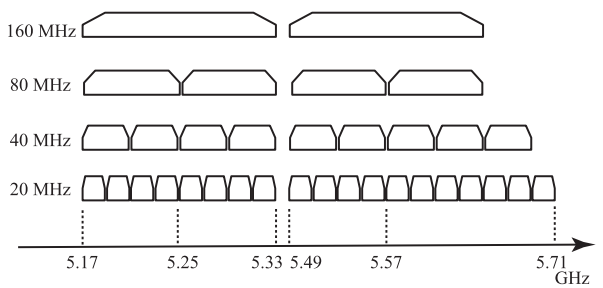

Fig. 1 Available frequency band and channel arrangement in Japan.

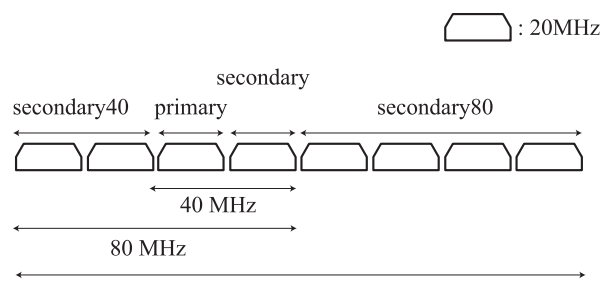

$160 \mathrm{MHz}$ or $80 \mathrm{MHz}+80 \mathrm{MHz}$

Fig. 2 Example of primary, secondary, secondary 40 , and secondary 80 channels in the frequency range from $5.17 \mathrm{GHz}$ to $5.33 \mathrm{GHz}$. second $20 \mathrm{MHz}$ channels are set as the secondary 40 channel, and the fifth, sixth, seventh, and eighth $20 \mathrm{MHz}$ channels are set as the secondary 80 channel to be aggregated to the $80 \mathrm{MHz}$ and $160 \mathrm{MHz}$ channels.

\subsection{Game Theory [10]-[12]}

Game theory is used for the mathematical analysis of strategic interaction. A game is a formal representation of a situation in which a number of individuals interact with each other in a strategically interdependent setting. This means that each individual's welfare depends not only on his/her own actions but also on the actions of the other individuals.

In what follows, we explain the definition of a strategicform game. In addition, the concept of best-response correspondence, Nash equilibrium, and Price of Anarchy (PoA) [10] are presented.

\subsubsection{Strategic-Form Games}

A game in a strategic-form has three elements: the set of players $\mathcal{N}=\{1, \ldots, N\}$, which represents the decisionmaking entities in the assumed situation; the pure-strategy space $\mathcal{A}_{i}$, which represents possible actions of each player; and the utility function $u_{i}$, which represents the profit of each player as a result of decision making, where $i \in \mathcal{N}$ represents the index of players. Formally, we represent a game as $G=\left(\mathcal{N}, \mathcal{A},\left\{u_{i}\right\}_{i \in \mathcal{N}}\right)$, where $\mathcal{A}=\left\{\mathcal{A}_{1} \times \cdots \times \mathcal{A}_{N}\right\}$ represents the set of pure-strategy spaces of all players.

We introduce the game-theoretic expressions. The strategies of all players is denoted by $\boldsymbol{a} \in \mathcal{A}$, the strategies excluding player $i$ is denoted by $\boldsymbol{a}_{-i}=$ $\left(a_{1}, \ldots, a_{i-1}, a_{i+1}, \ldots, a_{N}\right)$.

\subsubsection{Best-Response Correspondence}

We can say that player $i$ 's best-response correspondence $b_{i}$ : $\mathcal{A}_{-i} \rightarrow \mathcal{A}_{i}$ in the game $\left(\mathcal{N}, \mathcal{A},\left\{u_{i}\right\}_{i \in \mathcal{N}}\right)$, is the correspondence that assigns the set

$$
\begin{aligned}
b_{i}\left(\boldsymbol{a}_{-i}\right)=\left\{a_{i}^{\star} \in\right. & \mathcal{A}_{i}: \\
& \left.u_{i}\left(a_{i}^{\star}, \boldsymbol{a}_{-i}\right) \geq u_{i}\left(a_{i}, \boldsymbol{a}_{-i}\right), \forall a_{i} \in \mathcal{A}_{i}\right\}
\end{aligned}
$$

to each $\boldsymbol{a}_{-i} \in \mathcal{A}_{-i}$.

\subsubsection{Nash Equilibrium}

With the notion of best-response correspondence, we define Nash equilibrium as follows: the strategy profile $\left(a_{1}^{\star}, \ldots, a_{N}^{\star}\right)$ is the Nash equilibrium of game $G=$ $\left(\mathcal{N}, \mathcal{A},\left\{u_{i}\right\}_{i \in \mathcal{N}}\right)$ if and only if $a_{i}^{\star} \in b_{i}\left(\boldsymbol{a}_{-i}^{\star}\right), \forall i$. Let the set of strategies in Nash equilibria of game $G$ be denoted by a subset $\mathcal{E}(G) \subseteq \mathcal{A}$. In Nash equilibrium, neither player has motivation to change his/her strategy unilaterally to improve his/her utility. Thus, Nash equilibrium can be viewed as an operation point of decentralized control, and we discuss the throughput performance of Nash equilibria. 


\subsubsection{Price of Anarchy}

PoA is a performance measure in game theory, which represents how the efficiency of a system degrades owing to the selfish behavior of players compared to the optimal performance [10]. PoA is defined as the ratio between the worst equilibrium and the optimal centralized solution in terms of the aggregated utility as follows:

$$
\operatorname{PoA}(G)=\frac{\max _{\boldsymbol{a} \in \mathcal{A}} \sum_{i \in \mathcal{N}} u_{i}(\boldsymbol{a})}{\min _{\boldsymbol{a} \in \mathcal{E}(G)} \sum_{i \in \mathcal{N}} u_{i}(\boldsymbol{a})} .
$$

PoA has a value greater than or equal to 1 . When PoA is close to 1 , decentralized decision-making can be used to achieve performance similar to centralized decisionmaking.

\section{System Model}

\subsection{Location and Number of APs and Stations}

For ease of analysis, we assume the following simple scenario: there are three APs 1, 2, and 3, and all stations are within the overlap of all cells formed by these APs, as shown in Fig. 3, where cell $i$ represents the cell formed by AP $i$. Thus, all APs and stations are able to sense the frames transmitted from all other APs and stations. Note another scenario in which there are more than three APs is discussed in Sect. 6.3. In addition, we assume that traffic between an AP to the stations in either direction is saturated, and there is no interference between neighboring channels.

The reason why we assume three APs is the purpose of this paper, i.e., to know the efficiency of coordination of the APs when there remains an uncontrollable AP. In particular, three is the minimum number required to discuss coordinated APs and an uncontrollable AP. In other words, we assume two AP groups: the first group consists of two APs, and the second group consists of one AP.

We would like to emphasize that the assumed scenario is the minimum one to consider the coordination of APs and uncontrollable APs.

To evaluate the effectiveness of coordinating APs, we introduce "independent APs scenario" and "coordinated APs scenario". In the independent APs scenario, each of the three APs independently selects channels with the purpose of maximizing its own throughput. In the coordinated APs scenario, a centralized controller coordinates APs 1 and 2, which jointly select channels to maximize their total throughput, whereas AP 3 independently selects a channel to maximize its own throughput.

\subsection{Available Frequency Bands and Channels}

Each AP selects a channel from the following three types

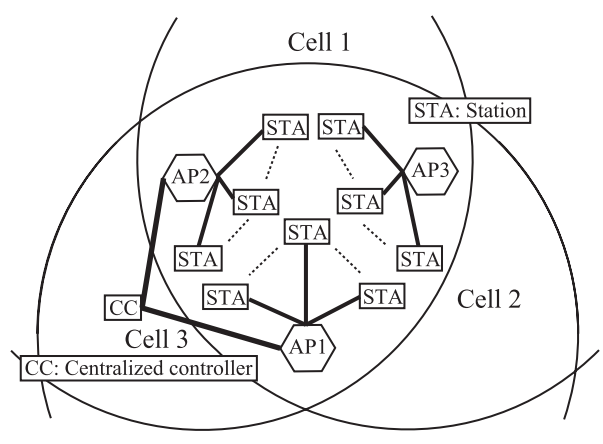

Fig. 3 Overlapping cells formed by three APs.

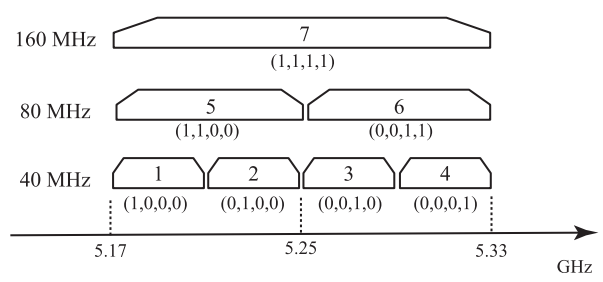

Fig. 4 Assumed multibandwidth channels.

of bandwidth channels: $40 \mathrm{MHz}, 80 \mathrm{MHz}$, and $160 \mathrm{MHz}$. Here, we exclude the $20 \mathrm{MHz}$ channel without loss of generality, because it is sufficient for us to consider at least three orthogonal channels for three APs. Thus, we consider four $40 \mathrm{MHz}$ orthogonal channels from $5.17 \mathrm{GHz}$ to $5.33 \mathrm{GHz}$. We use the channel index from 1 to 7 as shown in Fig. 4. For mathematical expressions, we use another expression for the channel index; for example, channel 6 is represented by $(0,0,1,1)$, where " 0 " represents an idle $40 \mathrm{MHz}$ channel and "1" represents a busy $40 \mathrm{MHz}$ channel. For example, when AP 1 selects channel 6, the strategy of AP 1 is $a_{1}=(0,0,1,1)$.

\section{Game-theoretic Formulation of Multibandwidth Channel Selection}

\subsection{Independent APs Scenario}

The independent APs scenario introduced in Sect. 3.1, i.e., three APs independently select channels to maximize their own throughput, can be formulated as a strategic-form game, where the set of APs $\mathcal{N}_{\mathrm{I}}=\{1,2,3\}$ is the set of players. The sets of available multibandwidth channels are the strategy spaces. In this case, the pure-strategy space of player $i$ can be written as

$$
\begin{array}{r}
\mathcal{A}_{i}=\{(1,0,0,0),(0,1,0,0),(0,0,1,0),(0,0,0,1), \\
(1,1,0,0),(0,0,1,1),(1,1,1,1)\}
\end{array}
$$

using the channel index expression defined in Sect.3.2. In addition, the expected values of the throughput are the utilities. Note that the throughput of AP $i$ depends on not only the channel of AP $i$ but also the channels selected by the other APs, i.e., the utility of AP $i$ is represented by $u_{i}(\boldsymbol{a})$ where $\boldsymbol{a}=\left(a_{1}, a_{2}, a_{3}\right)$. This game-theoretic formulation is 
summarized as a game $G_{I}=\left(\mathcal{N}_{\mathrm{I}}, \mathcal{A}_{1} \times \mathcal{A}_{2} \times \mathcal{A}_{3},\left\{u_{1}, u_{2}, u_{3}\right\}\right)$. Note that all factors of $G_{\mathrm{I}}$ are assumed to be known by all the APs.

\subsection{Coordinated APs Scenario}

In the coordinated APs scenario, a centralized controller coordinates APs 1 and 2, and it selects both channels of APs 1 and 2 to maximize their aggregated throughput, whereas AP 3 independently selects a channel to maximize its own throughput. Similar to the independent APs scenario, we formulate this situation as a strategic-form game. Here, let a centralized controller for APs 1 and 2 be denoted by player 12, and AP 3 is player 3 . Let the set of players in this scenario be denoted by $\mathcal{N}_{\mathrm{C}}=\{12,3\}$. In this case, the pure-strategy space of player 12 , is $\mathcal{A}_{12}=\mathcal{A}_{1} \times \mathcal{A}_{2}$, which means that the pure-strategy space is expanded as compared to that of the independent APs scenario. The utility of player 12 is set to the total throughput of APs 1 and 2; thus, $u_{12}\left(a_{12}, a_{3}\right)=u_{1}\left(a_{1}, a_{2}, a_{3}\right)+u_{2}\left(a_{1}, a_{2}, a_{3}\right)$. This game-theoretic formulation is summarized as a game $G_{\mathrm{C}}=\left(\mathcal{N}_{\mathrm{C}}, \mathcal{A}_{12} \times \mathcal{A}_{3},\left\{u_{12}, u_{3}\right\}\right)$. As in the independent APs scenario, all factors of $G_{\mathrm{C}}$ are assumed to be known by all the APs and the centralized controller.

\section{Utility Function}

In this section, we describe the details of the utility function. Because the value of the utility function is the expected value of throughput, the details of channel arrangement, data rate, and carrier sense multiple access with collision avoidance (CSMA/CA) are discussed.

\subsection{Channel Arrangement}

\subsubsection{Primary Channel}

The channel arrangement of a multibandwidth channel was introduced in Sect. 2.1. In addition, there are two restrictions for the use of multibandwidth channels in IEEE 802.11ac [2]. The first restriction is where each AP needs to use continuous idle channels. Here, we consider a case where a channel selected by an AP is completely or partially overlapped by other APs. For example, we assume a scenario wherein an AP selects a $160 \mathrm{MHz}$ channel, and the primary, secondary, and secondary 80 channels are idle, while the secondary 40 channel is busy. In this case, the AP can only use the primary and secondary channel as a $40 \mathrm{MHz}$ bandwidth channel, but the AP cannot use secondary40 and secondary 80 .

The second restriction is related to the setting of the primary channel. As mentioned in Sect. 2.1, in IEEE 802.11ac, each AP should set one $20 \mathrm{MHz}$ channel as a primary channel, regardless of the bandwidth of the communication channel. In addition, if the channels selected by multiple APs completely or partially overlap, these APs should set the same $20 \mathrm{MHz}$ channel as the primary channel.
However, even if we adopt the aforementioned primary channel setting, it is possible that the primary channel cannot be determined uniquely. When there are two orthogonal channels selected by APs $j$ and $k$ in a channel with wider bandwidth selected by AP $i$, AP $i$ should set its primary channel in accordance with the primary channel of APs $j$ or $k$. However it is not clearly defined as to which primary channels should be selected by AP $i$. In this paper, we assume that APs 1 and 2 are coordinated; thus, in this situation, it is assumed that APs 1 and 2 set the same primary channel.

\subsubsection{Equivalent Channel Assignment}

Particularly in this situation, we assume that a given AP cannot use channels occupied by other APs whose primary channels are different from their own primary channel. The reason is as follows. For example, we consider the channel selection shown in Fig. 5. APs 2 and 3 select different $80 \mathrm{MHz}$ channels overlapping with the $160 \mathrm{MHz}$ channel selected by AP 1 . Here, the primary channels of APs 1 and 2 are set to be the same, while the primary channel of AP 3 differs from APs 1 and 2. In the case of saturated traffic, these channels are rarely idle simultaneously. In particular, because AP 1 needs carrier sense of primary, secondary, and secondary 40 in turn before secondary 80 , which would be frequently occupied by AP 3, the secondary 80 channel can rarely be used by AP 1 .

In this case, we assume that the throughput performance of the channel selection for AP 1 in Fig. 5, $a_{1}=$ $(1,1,1,1)$, is equivalent to the performance of channel selection shown in Fig. 6, $a_{1}^{\prime}=(1,1,0,0)$. Here, we denote the channel assignment of AP $i$ to be $a_{i}$, and the equivalent channel assignment to be $a_{i}^{\prime}$. Note that this assumption is required for ease of link-level throughput evaluation. Using

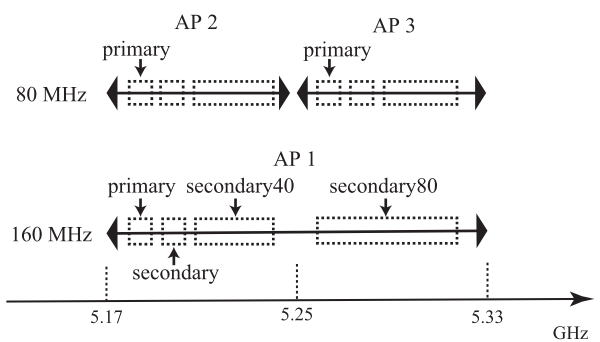

Fig. 5 Example of channel arrangement.

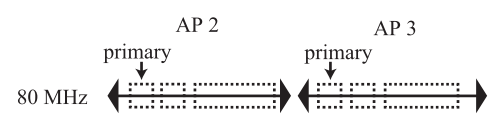

AP 1

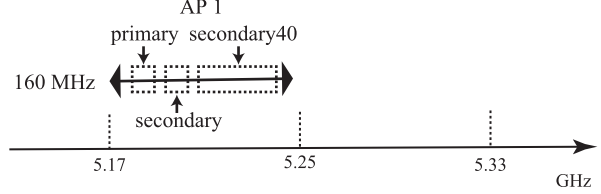

Fig. 6 Equivalent channel arrangement of Fig. 5. 
Table 1 Data rate when the received power is $-60 \mathrm{dBm}$.

\begin{tabular}{cccc}
\hline $\begin{array}{c}\text { Channel } \\
\text { bandwidth }\end{array}$ & $\begin{array}{c}\text { Data } \\
\text { rate }\end{array}$ & Modulation & $\begin{array}{c}\text { Coding } \\
\text { rate }\end{array}$ \\
\hline $40 \mathrm{MHz}$ & $270 \mathrm{Mbit} / \mathrm{s}$ & $64 \mathrm{QAM}$ & $5 / 6$ \\
$80 \mathrm{MHz}$ & $468 \mathrm{Mbit} / \mathrm{s}$ & $64 \mathrm{QAM}$ & $2 / 3$ \\
$160 \mathrm{MHz}$ & $702 \mathrm{Mbit} / \mathrm{s}$ & $16 \mathrm{QAM}$ & $3 / 4$ \\
\hline
\end{tabular}

this assumption, we can estimate the throughput as shown in Sect. 5.3.

\subsection{Data Rate}

For ease of analysis, the received power at each station and AP is assumed to be the same. Thus, the data rate only depends on the channel bandwidth. Unless otherwise stated, the received power is set to be $-60 \mathrm{dBm}$. The guard interval is set to be $800 \mathrm{~ns}$, independent of the received power, and the number of streams is set to two. The modulation and coding scheme (MCS) depending on the channel bandwidth and resultant data rate are summarized in Table 1. Even if we use the $80 \mathrm{MHz}$ channel instead of the $40 \mathrm{MHz}$ channel, we achieve less than double the data rate of the $40 \mathrm{MHz}$ channel because the transmit power is fixed, independent of the channel bandwidth. Thus, when the channel bandwidth is doubled, the received power density decreases. In this case, the receiver sensitivity for each MCS should be increased by $3 \mathrm{~dB}$ as described in [3].

\subsection{Impact of the Number of Stations and Frame Length on the Throughput}

We explain how to estimate the throughput with the CSMA/CA protocol. For the ease of analysis, RTS/CTS is not considered.

When each AP selects orthogonal channels, they do not suffer from inter-cell contention. On the other hand, when some APs select the same channels or a part of the selected channels overlaps, they suffer from inter-cell contention. The interference between channels at different frequencies is assumed to be negligible.

Let the number of transmitters related to cell $i$, i.e., AP $i$ itself and the stations associated with AP $i$, be denoted by $m_{i}$. For example, if five stations are associated with AP 1 , $m_{1}=6$. In addition, let the number of transmitters in cell $i$ using the same channel of AP $i$ be denoted by $l_{i}(\boldsymbol{a})$, which is a function of the selected channel as

$$
l_{i}\left(\boldsymbol{a}^{\prime}\right)=\sum_{j \in \mathcal{N} \backslash\{i\}} m_{j} H_{0}\left(a_{i}^{\prime} a_{j}^{\prime \mathrm{T}}\right),
$$

where superscript $\mathrm{T}$ represents transposition and $H_{0}(t)$ denotes the Heaviside step function; $H_{0}(t)=1(t>0), H_{0}(t)=$ $0(t \leq 0)$. Note that $a_{i}^{\prime} a_{j}^{\prime \mathrm{T}}=0$ when APs $i$ and $j$ select orthogonal channels, and $a_{i}^{\prime} a_{j}^{\mathrm{T}}>0$ otherwise.

\subsubsection{Fixed Frame Length Case}

Here, we conduct performance evaluations under two different assumptions of the frame length in the time domain,
Table 2 Relationship between data rate and frame length $(-60 \mathrm{dBm})$.

\begin{tabular}{ccc}
\hline $\begin{array}{c}\text { Channel } \\
\text { bandwidth }\end{array}$ & $\begin{array}{c}\text { Data } \\
\text { rate }\end{array}$ & $\begin{array}{c}\text { Payload } \\
\text { size }\end{array}$ \\
\hline $40 \mathrm{MHz}$ & $270 \mathrm{Mbit} / \mathrm{s}$ & $25000 \mathrm{~B}$ \\
$80 \mathrm{MHz}$ & $468 \mathrm{Mbit} / \mathrm{s}$ & $43300 \mathrm{~B}$ \\
$160 \mathrm{MHz}$ & $702 \mathrm{Mbit} / \mathrm{s}$ & $65000 \mathrm{~B}$ \\
\hline
\end{tabular}

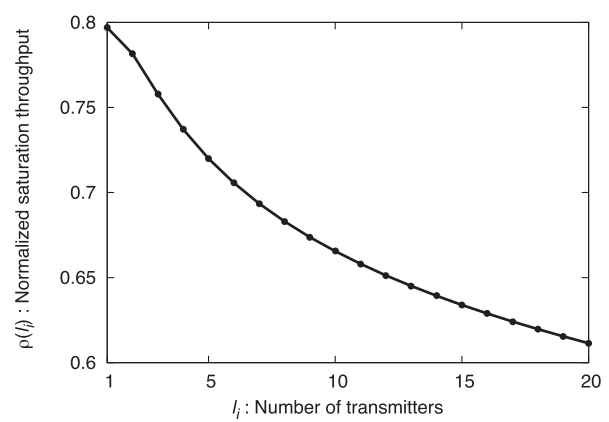

Fig. 7 Normalized saturation throughput versus the number of transmitters (FFL case).

i.e., the fixed frame length (FFL) case and variable frame length (VFL) case. For ease of analysis in FFL case, the payload size, i.e., MAC Service Data Unit (MSDU) size, in bits is changed so that the frame length in the time domain is fixed, as shown in Table 2, even when the channel bandwidth is changed, and thus, the data rate is changed. In this case, the saturation throughput normalized by the data rate is the same regardless of the data rate, where the saturation throughput is defined as the total throughput under saturated traffic as defined in [13]. The relationship between the normalized saturation throughput and the number of transmitters is presented in [13] and shown in Fig. 7, where $\rho\left(l_{i}\right)$ represents the normalized saturation throughput depending on the number of transmitters using the same channel with $\mathrm{AP} i, l_{i}$. We can see that the saturation throughput decreases with an increase in the number of transmitters [13].

Let the data rate when AP $i$ chooses channel $a_{i}^{\prime}$ be denoted by $R_{a_{i}^{\prime}} \in\{270 \mathrm{Mbit} / \mathrm{s}, 468, \mathrm{Mbit} / \mathrm{s}, 702 \mathrm{Mbit} / \mathrm{s}\}$, and the frame length in the time domain be denoted by $T_{i}$. Using these expressions, the throughput of cell $i$ when APs $i, j$, and $k$ select an overlapping channel is calculated as

$$
u_{i}(\boldsymbol{a})=R_{a_{i}^{\prime}} \cdot \rho\left(l_{i}\left(\boldsymbol{a}^{\prime}\right)\right) \cdot \frac{m_{i} T_{i}}{m_{i} T_{i}+m_{j} T_{j}+m_{k} T_{k}} .
$$

Moreover, because the frame length is assumed to be the same at any data rate, i.e., $T_{i}=T_{j}=T_{k}$, (5) can be written as

$$
u_{i}(\boldsymbol{a})=R_{a_{i}^{\prime}} \cdot \rho\left(l_{i}\left(\boldsymbol{a}^{\prime}\right)\right) \cdot \frac{m_{i}}{l_{i}\left(\boldsymbol{a}^{\prime}\right)} .
$$

Note that (6) can also be used when the channels are not overlapped. In addition, note that a similar expression to (6) has been presented in [6], but lacks the multibandwidth factor.

For example, we calculate the throughput considering $a_{1}=(1,1,1,1), a_{2}=(1,1,0,0), a_{3}=(0,0,1,1)$ as 
shown in Fig. 5. First, as discussed in Sect. 5.1, the channel arrangement of each AP is equivalently treated as $a_{1}^{\prime}=$ $(1,1,0,0), a_{2}^{\prime}=(1,1,0,0), a_{3}^{\prime}=(0,0,1,1)$ as shown in Fig. 6. Then, from (6),

$$
u_{1}(\boldsymbol{a})=468 \mathrm{Mbit} / \mathrm{s} \cdot 0.65 \cdot \frac{6}{12}=152 \mathrm{Mbit} / \mathrm{s} \text {. }
$$

In the same manner, $u_{2}(\boldsymbol{a})=152 \mathrm{Mbit} / \mathrm{s}$ and $u_{3}(\boldsymbol{a})=$ $330 \mathrm{Mbit} / \mathrm{s}$.

\subsubsection{Variable Frame Length Case}

In the variable frame length (VFL) case, the payload size is always set to $65000 \mathrm{~B}$, regardless of the channel bandwidth, and the frame length in the time domain changes depending on the data rate. In this case, not only $R_{a_{i}^{\prime}}$ but also $\rho\left(l_{i}\left(\boldsymbol{a}^{\prime}\right)\right)$ depend on the data rate; thus, we are not able to separate the saturation throughput according to $R_{a_{i}^{\prime}}$ and $\rho\left(l_{i}\left(\boldsymbol{a}^{\prime}\right)\right)$, as in the FFL case. Instead, we calculate $u_{i}$ using the expression of throughput [13] considering different frame lengths in the time domain, assuming that the collision probability of frames with greater than or equal to three different data rates is small enough to be ignored.

\section{Numerical Evaluations}

In this section, we discuss Nash equilibria by numerical evaluations. The focus here is not to increase the total throughput of all APs but to increase the total throughput of the coordinated APs. Unless otherwise stated, five stations are associated with each AP, i.e., $m_{1}=6, m_{2}=6$, and $m_{3}=6$. The channel arrangements of each AP at Nash equilibria are summarized in Table 3 , and the throughput is shown in Fig. 8. Note that we excluded redundant channel selections considering the symmetric properties of the channel. These equilibria are found such that the throughput of all channel arrangements is first evaluated, and then Nash equilibria are found by exhaustive search. To draw Fig. 8, we calculated the throughput of all overlapping channel patterns according to the data rates of each AP and station, and then plotted the throughput in Fig. 8. Nash equilibria in game $G_{\mathrm{I}}$ are represented by $\times$, and those in game $G_{\mathrm{C}}$ are represented by $\circ$. The other points are represented by + .

\subsection{Channel Arrangements at Nash Equilibria}

Here, we discuss the channel arrangements at Nash equilibria. By comparing the channel arrangements in the individual and coordinated APs scenarios, i.e., comparing Nash equilibria of game $G_{\mathrm{I}}$ and those of game $G_{\mathrm{C}}$, we demonstrate that the coordination of APs is an effective way to deviate from an undesirable Nash equilibrium, at which all APs occupies the same $160 \mathrm{MHz}$ channel and they suffer from severe contention.

\subsubsection{Nash Equilibria in Game $G_{\mathrm{I}}$}

In game $G_{\mathrm{I}}$, there are 14 Nash equilibria summarized in Ta-
Table 3 Remarkable channel selections in Figs. 8 to 12.

\begin{tabular}{c|ccc}
\hline & \multicolumn{3}{|c}{ Channel arrangement } \\
Index & $a_{1}$ & $a_{2}$ & $a_{3}$ \\
\hline $\mathrm{A}$ & $(1,1,0,0)$ & $(0,0,1,0)$ & $(0,0,0,1)$ \\
$\mathrm{B}$ & $(1,1,0,0)$ & $(0,0,0,1)$ & $(0,0,1,0)$ \\
$\mathrm{C}$ & $(1,0,0,0)$ & $(0,0,1,1)$ & $(0,1,0,0)$ \\
$\mathrm{D}$ & $(0,1,0,0)$ & $(0,0,1,1)$ & $(1,0,0,0)$ \\
$\mathrm{E}$ & $(0,1,0,0)$ & $(1,0,0,0)$ & $(0,0,1,1)$ \\
$\mathrm{F}$ & $(1,0,0,0)$ & $(0,1,0,0)$ & $(0,0,1,1)$ \\
$\mathrm{G}$ & $(1,1,0,0)$ & $(0,0,1,1)$ & $(0,0,1,1)$ \\
$\mathrm{H}$ & $(1,1,0,0)$ & $(1,1,0,0)$ & $(0,0,1,1)$ \\
$\mathrm{I}$ & $(1,1,0,0)$ & $(1,1,1,1)$ & $(0,0,1,1)$ \\
$\mathrm{J}$ & $(1,1,1,1)$ & $(1,1,0,0)$ & $(0,0,1,1)$ \\
$\mathrm{K}$ & $(1,1,0,0)$ & $(0,0,1,1)$ & $(1,1,0,0)$ \\
$\mathrm{L}$ & $(1,1,1,1)$ & $(0,0,1,1)$ & $(1,1,0,0)$ \\
$\mathrm{M}$ & $(1,1,0,0)$ & $(0,0,1,1)$ & $(1,1,1,1)$ \\
$\mathrm{N}$ & $(1,1,1,1)$ & $(1,1,1,1)$ & $(1,1,1,1)$ \\
\hline
\end{tabular}

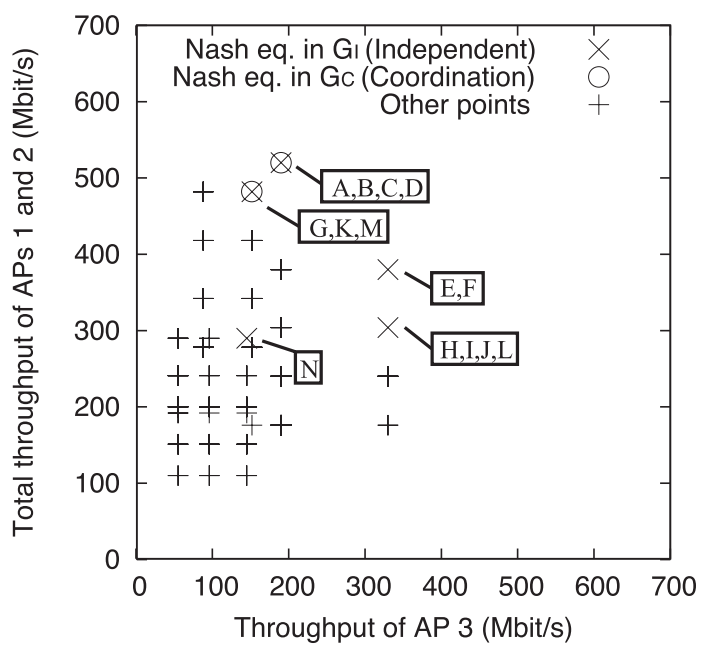

Fig. 8 Throughput (FFL case, $-60 \mathrm{dBm}, m_{1}=6, m_{2}=6, m_{3}=6$ ).

ble 3 represented by $\times$ in Fig. 8. At all of these Nash equilibria, the available frequency bands shown in Fig. 4 are completely used by three APs, and the channel arrangements are broadly divided into three patterns. Here, we discuss these three patterns one by one.

In the first pattern at Nash equilibrium $\mathrm{N}$, all APs select the same $160 \mathrm{MHz}$ channel, which causes severe contention among APs and stations, and the throughput is degraded. In the second pattern at Nash equilibria A, B, C, D, E, and F, each AP selects an orthogonal channel, and thus, contention does not occur among the APs. In the third pattern, at Nash equilibria G, H, I, J, L, M, and K, all APs select a $80 \mathrm{MHz}$ channel, and the channels of two APs overlap.

\subsubsection{Nash Equilibria in Game $G_{\mathrm{C}}$}

In game $G_{\mathrm{C}}$, there are seven Nash equilibria represented by ○ in Fig. 8. Compared to game $G_{\mathrm{I}}$, points E, F, H, I, J, L, and $\mathrm{N}$ are no longer Nash equilibria in game $G_{\mathrm{C}}$

At point $\mathrm{N}$ in game $G_{\mathrm{I}}$, APs 1,2 , and 3 do not have a motivation to change their channel unilaterally. This is because if one AP changes its channel from the $160 \mathrm{MHz}$ 
channel to a $80 \mathrm{MHz}$ channel, the $80 \mathrm{MHz}$ channel is still overlapped by other APs and thus the AP cannot increase its throughput. Note even in this situation, the channels selected by all APs still partially overlap, thus all APs set the same $20 \mathrm{MHz}$ channel as the primary channel as discussed in Sect. 5.1.1.

In contrast, in game $G_{\mathrm{C}}$, the centralized controller can change both channels of APs 1 and 2 at the same time, and thus has a motivation to change the channels of APs 1 and 2 from the same $160 \mathrm{MHz}$ channel 7 to different $80 \mathrm{MHz}$ channels 5 and 6, resulting in Nash equilibrium $M$. This means that APs can deviate from undesirable Nash equilibrium N. This important mechanism is derived in the same way as the equivalent channel assignment discussed in Sect. 5.1.2, i.e., even when AP 3 chooses the $160 \mathrm{MHz}$ channel, AP 3 equivalently uses $80 \mathrm{MHz}$ as the consequence of the channel assignment of the centralized controller.

In the same way, other excluded points can be explained. At points $\mathrm{E}$ and $\mathrm{F}$ in $G_{\mathrm{I}}$, neither $\mathrm{AP} 1,2$, or 3 has motivation to change its channel. In contrast, the centralized controller in game $G_{\mathrm{C}}$ has motivation to change the channels of APs 1 and 2 from different $40 \mathrm{MHz}$ channels 1 and 2 to different $80 \mathrm{MHz}$ channels 5 and 6 , resulting in Nash equilibrium G. From points H, I, and J, the centralized controller also changes these channels and results in Nash equilibrium G. From point L, the centralized controller changes the channel of AP 1, yielding Nash equilibrium K.

\subsubsection{Comparison of Games $G_{\mathrm{I}}$ and $G_{\mathrm{C}}$}

In game $G_{\mathrm{I}}$, three APs independently select channels, in which each AP selects a channel to maximize its own throughput. As a result, the low total throughput points of APs 1 and 2 also become Nash equilibria. On the other hand, in game $G_{\mathrm{C}}$, APs 1 and 2 are coordinated, where APs 1 and 2 jointly select their channels to maximize the total throughput of APs 1 and 2, while AP 3 selects a channel to maximize its own throughput. Therefore, APs 1 and 2 can only choose strategies that increase the total throughput of APs 1 and 2. As a result, the high total throughput points of APs 1 and 2 become Nash equilibria.

Here, we define the throughput improvement ratio (TIR) through coordination as follows:

$$
\mathrm{TIR}=\frac{\min _{\boldsymbol{a} \in \mathcal{E}\left(G_{\mathrm{C}}\right)} \sum_{i \in\{1,2\}} u_{i}(\boldsymbol{a})}{\min _{\boldsymbol{a} \in \mathcal{E}\left(G_{\mathrm{I}}\right)} \sum_{i \in\{1,2\}} u_{i}(\boldsymbol{a})} .
$$

In this case, $\mathrm{TIR}=1.66$.

Here, we evaluate the PoA of Fig. 8. In game $G_{\mathrm{I}}$, $\operatorname{PoA}\left(G_{\mathrm{I}}\right)=1.63$. On the contrary, in game $G_{\mathrm{C}}, \operatorname{PoA}\left(G_{\mathrm{C}}\right)=$ 1.12. From this result, we can say that coordinating APs dramatically decreases PoA, i.e., even with decentralized control, the performance of game $G_{\mathrm{C}}, \min _{\boldsymbol{a} \in \mathcal{E}\left(G_{\mathrm{C}}\right)}\left(u_{12}(\boldsymbol{a})+u_{3}(\boldsymbol{a})\right)$, is similar to the performance of centralized control of APs 1 ,
2, and $3, \max _{\boldsymbol{a} \in \mathcal{A}} \sum_{i \in \mathcal{N}_{\mathrm{I}}} u_{i}(\boldsymbol{a})$. In other words, even if we could not control all of the APs, control of a part of the APs has the potential to substantially decrease PoA.

\subsection{Comparison to Cases with Different Parameters}

Here, we treat the case of Fig. 8 as the standard case. By comparing the standard case to the other cases, we clarify that the coordination of APs is an effective method to increase throughput independent of parameters.

\subsubsection{Comparison between the FFL and VFL Cases}

The throughput of the VFL case is shown in Fig. 9, while Fig. 8 shows the performance for the FFL case. Although the value of throughput at each channel arrangement differs between the FFL and VFL cases, as shown in Figs. 8 and 9, the channel arrangements at Nash equilibria are the same. Thus, the effect of the change of frame length is smaller than that of channel arrangement. In Fig. 9, TIR $=1.72$. Therefore, in the following evaluations, we only consider the FFL case.

\subsubsection{Impact of Received Power}

Thus far, the received power is assumed to be $-60 \mathrm{dBm}$. To assess the impact of the received power on Nash equilibria, Fig. 10 shows the throughput at the received power setting of $-70 \mathrm{dBm}$.

In this case, the data rate summarized in Table 4 is used instead of Table 1. All other parameters are the same as those used in Fig. 8. By comparing Figs. 8 and 10, we can see that the number of Nash equilibria is the same, and the channel arrangements at Nash equilibria are also the same. Thus, the received power does not have a large impact on the channel arrangements at Nash equilibria. This is because the ratio of throughput increase to the change in channel

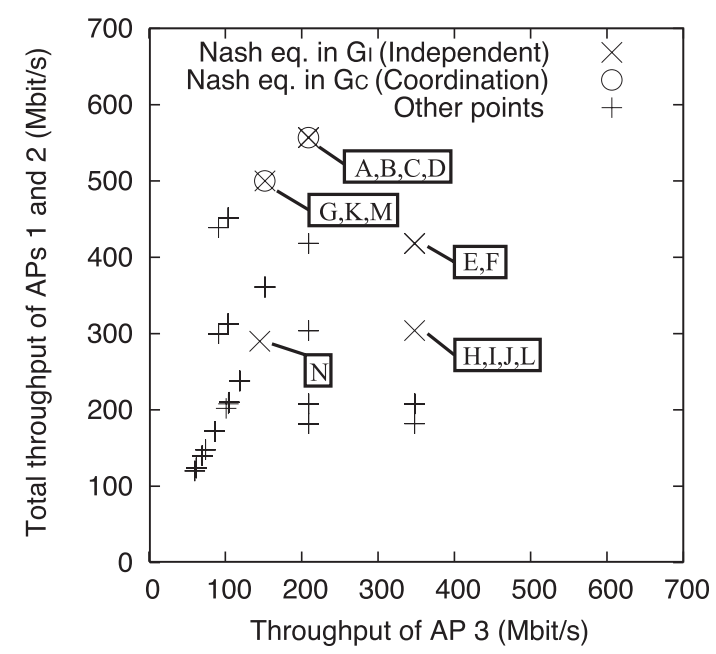

Fig. 9 Throughput (VFL case, $-60 \mathrm{dBm}, m_{1}=6, m_{2}=6, m_{3}=6$ ). 


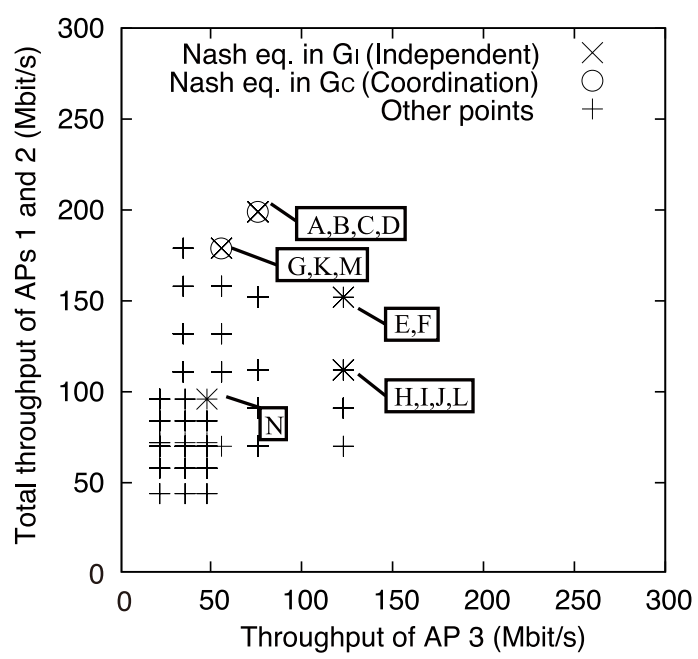

Fig. 10 Throughput (FFL case, $-70 \mathrm{dBm}, m_{1}=6, m_{2}=6, m_{3}=6$ ).

Table 4 Data rate when the received power is $-70 \mathrm{dBm}$.

\begin{tabular}{cccc}
\hline $\begin{array}{c}\text { Channel } \\
\text { bandwidth }\end{array}$ & $\begin{array}{c}\text { Data } \\
\text { rate }\end{array}$ & Modulation & $\begin{array}{c}\text { Coding } \\
\text { rate }\end{array}$ \\
\hline $40 \mathrm{MHz}$ & $108 \mathrm{Mbit} / \mathrm{s}$ & 16QAM & $1 / 2$ \\
$80 \mathrm{MHz}$ & $176 \mathrm{Mbit} / \mathrm{s}$ & QPSK & $3 / 4$ \\
$160 \mathrm{MHz}$ & $234 \mathrm{Mbit} / \mathrm{s}$ & QPSK & $1 / 2$ \\
\hline
\end{tabular}

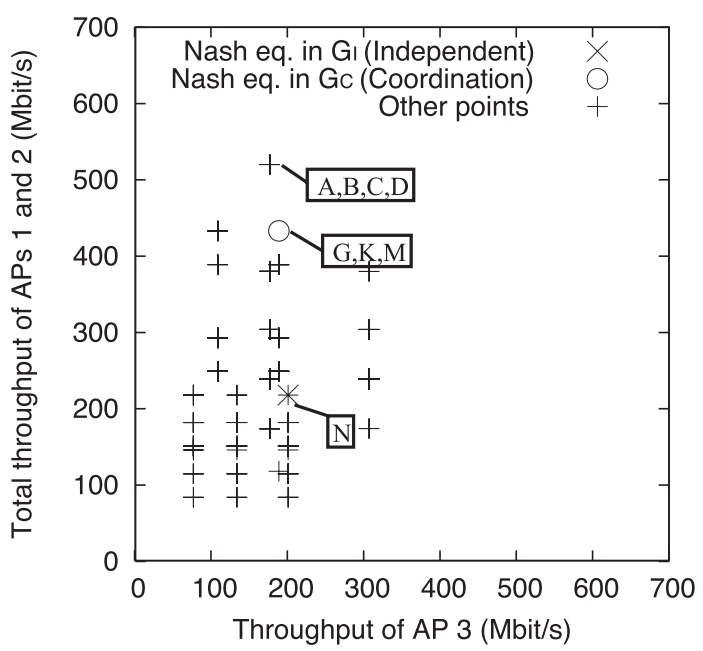

Fig. 11 Throughput (FFL case, $-60 \mathrm{dBm}, m_{1}=6, m_{2}=6, m_{3}=11$ ).

bandwidth is the same regardless of the received power.

\subsubsection{Impact of the Number of Stations}

Thus far, we have assumed that five stations are associated with each AP. To evaluate the impact of the number of associated stations, we study the following two cases. First, Fig. 11 shows the throughput assuming ten stations for AP 3 and five stations for both APs 1 and 2, i.e., $m_{1}=6, m_{2}=6$, and $m_{3}=11$. In comparison to Fig. 8, points A, B, C, and $\mathrm{D}$ are no longer Nash equilibria in both games $G_{\mathrm{I}}$ and $G_{\mathrm{C}}$. The reason is as follows. At these points, AP 3 uses the $40 \mathrm{MHz}$ channel. AP 3 cannot increase its throughput by

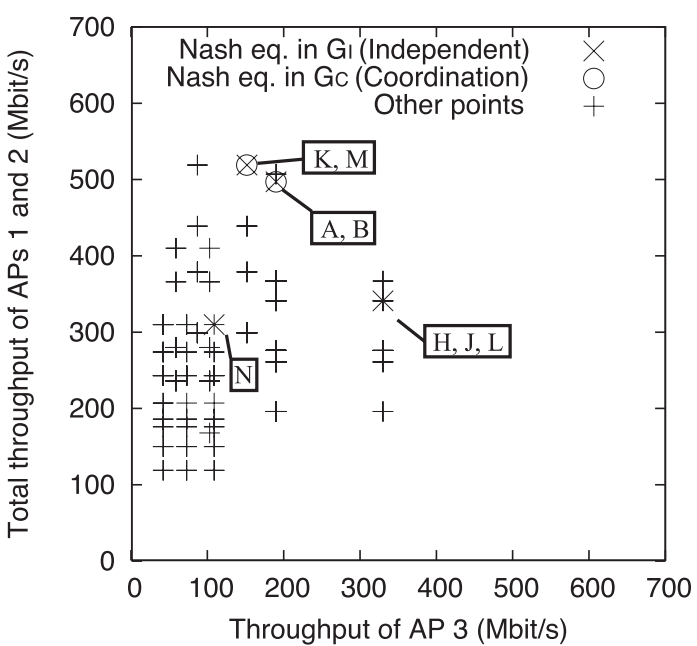

Fig. 12 Throughput (FFL case, $-60 \mathrm{dBm}, m_{1}=11, m_{2}=6, m_{3}=6$ ).

changing its channel bandwidth from $40 \mathrm{MHz}$ to $80 \mathrm{MHz}$ when $m_{3}=6$, while AP 3 can enhance its throughput when $m_{3}=11$. This is because the throughput of AP $3, u_{3}$, is increased by changing $m_{3}$ from 6 to 11 as seen in (6). By applying the best response in turn from these point when $m_{3}=11$, the selected channels converge to points $\mathrm{G}, \mathrm{K}$, and $\mathrm{M}$, which are the Nash equilibria.

PoA and TIR are evaluated as $\operatorname{PoA}\left(G_{\mathrm{I}}\right)=1.66$, $\operatorname{PoA}\left(G_{\mathrm{C}}\right)=1.12$, and TIR $=1.99$. Thus, even when $m_{3}=11$, the impact of the coordination of APs on PoA and TIR is still large.

Second, Fig. 12 shows the throughput assuming ten stations for AP 1 and five stations for APs 2 and 3, i.e., $m_{1}=11, m_{2}=6$, and $m_{3}=6$. In comparison to Fig. 8 , points $\mathrm{C}, \mathrm{D}$, and $\mathrm{G}$ are no longer Nash equilibria in both games $G_{\mathrm{I}}$ and $G_{\mathrm{C}}$. For point G, APs 1 and 2 are equivalent in terms of the total throughput of APs 1 and 2 when $m_{1}=m_{2}=6$; thus, points $\mathrm{G}$ and $\mathrm{K}$ are equivalent and are Nash equilibria. When these APs are no longer equivalent when $m_{1}=11$ and $m_{2}=6$, point $\mathrm{G}$ is excluded from Nash equilibria. Points $\mathrm{C}$ and $\mathrm{D}$ can be explained in a similar manner.

PoA and TIR are evaluated to be $\operatorname{PoA}\left(G_{\mathrm{I}}\right)=1.64$, $\operatorname{PoA}\left(G_{\mathrm{C}}\right)=1.02$, and TIR $=1.60$. Thus, even when $m_{1}=11$, the impact of the coordination of APs on PoA and TIR is still large. From these results, it is expected that the coordination of APs is an effective method for APs 1 and 2 to improve throughput at Nash equilibria regardless of the number of stations.

Finally, by comparing Figs. 11 and 12, we find that $\operatorname{PoA}\left(G_{\mathrm{C}}\right)$ in Fig. 12 is less than that in Fig. 11. Thus, if we can arbitrarily choose two APs from three APs to be managed through centralized control to minimize PoA, it is best to choose the AP that is associated with many stations.

6.3 Simplified Numerical Evaluations for More than Three APs

Thus far, we have assumed only three APs. In another sce- 
Table 5 Data rate when the received power is $-60 \mathrm{dBm}$.

\begin{tabular}{cccc}
\hline $\begin{array}{c}\text { Channel } \\
\text { bandwidth }\end{array}$ & $\begin{array}{c}\text { Data } \\
\text { rate }\end{array}$ & Modulation & $\begin{array}{c}\text { Coding } \\
\text { rate }\end{array}$ \\
\hline $20 \mathrm{MHz}$ & $156 \mathrm{Mbit} / \mathrm{s}$ & 256QAM & $3 / 4$ \\
$40 \mathrm{MHz}$ & $270 \mathrm{Mbit} / \mathrm{s}$ & 64QAM & $5 / 6$ \\
$80 \mathrm{MHz}$ & $468 \mathrm{Mbit} / \mathrm{s}$ & 64QAM & $2 / 3$ \\
$160 \mathrm{MHz}$ & $702 \mathrm{Mbit} / \mathrm{s}$ & 16QAM & $3 / 4$ \\
\hline
\end{tabular}

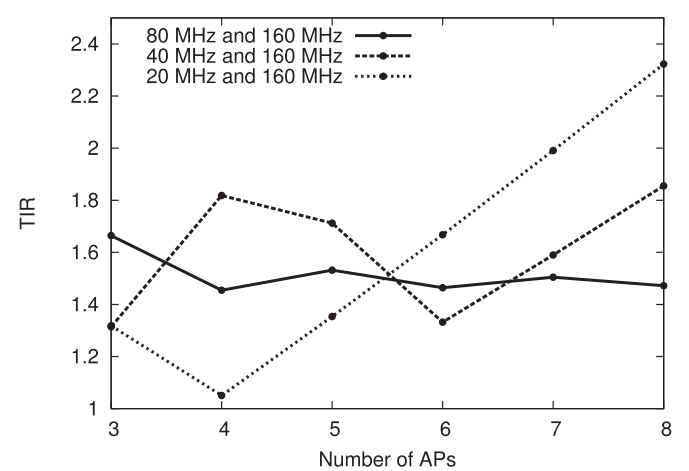

Fig. 13 Throughput improvement ratio for APs 1 and 2 through coordination.

nario in which there are more than three APs, throughput improvement through coordination of two APs should also be confirmed. For the ease of analysis, we decrease the degree of freedom, i.e., we restrict available channels for all APs to the following only two types of bandwidth channels: $160 \mathrm{MHz}$ and one narrower bandwidth. The narrower bandwidth is set to be $20 \mathrm{MHz}, 40 \mathrm{MHz}$, or $80 \mathrm{MHz}$. Note independent of the number of APs, the centralized controller coordinates only APs 1 and 2 as in previous sections.

When only $80 \mathrm{MHz}$ and $160 \mathrm{MHz}$ channels are available, two games $G_{\mathrm{I}}$ and $G_{\mathrm{C}}$ should be modified as

$$
\begin{aligned}
& \widetilde{G}_{\mathrm{I}}=\left(\mathcal{N}_{\mathrm{I}}, \widetilde{\mathcal{A}}_{1} \times \cdots \times \widetilde{\mathcal{A}}_{N},\left\{u_{1}, \ldots, u_{N}\right\}\right), \\
& \widetilde{G}_{\mathrm{C}}=\left(\mathcal{N}_{\mathrm{C}}, \widetilde{\mathcal{A}}_{12} \times \widetilde{\mathcal{A}}_{3} \times \cdots \times \widetilde{\mathcal{A}}_{N},\left\{u_{12}, u_{3}, \ldots, u_{N}\right\}\right),
\end{aligned}
$$

$$
\begin{aligned}
& \mathcal{N}_{\mathrm{I}}=\{1, \ldots, N\}, \\
& \mathcal{N}_{\mathrm{C}}=\{12,3, \ldots, N\}, \\
& \widetilde{\mathcal{P}}_{i}=\{(1,1,0,0),(0,0,1,1),(1,1,1,1)\},
\end{aligned}
$$

where $N$ represents the number of APs. Note when $N=3$, the difference between $G_{I}$ and $\widetilde{G}_{I}$ is the strategy space. When only $40 \mathrm{MHz}$ and $160 \mathrm{MHz}$ are available, the strategy space of games $\widetilde{G}_{\mathrm{I}}$ and $\widetilde{G}_{\mathrm{C}}$ should be modified as $\widetilde{\mathcal{A}}_{i}$ $=\{(1,0,0,0),(0,1,0,0),(0,0,1,0),(0,0,0,1),(1,1,1,1)\}$. When only $20 \mathrm{MHz}$ and $160 \mathrm{MHz}$ are available, the strategy space $\widetilde{\mathcal{A}}_{i}$ should be modified. Note the channel index expression defined in Sect. 3.2 cannot be used in this case, thus we omit the detailed expression of $\widetilde{\mathcal{A}}_{i}$ but numerical evaluation is possible. The MCS and data rate depending on the channel bandwidth are summarized in Table 5 .

TIR is shown in Fig. 13. Note when the number of APs is three, TIR with $80 \mathrm{MHz}$ and $160 \mathrm{MHz}$ channels is the same as TIR with $40 \mathrm{MHz}, 80 \mathrm{MHz}$, and $160 \mathrm{MHz}$ channels evaluated in Sect. 6.1.3. In all cases, TIR is greater than

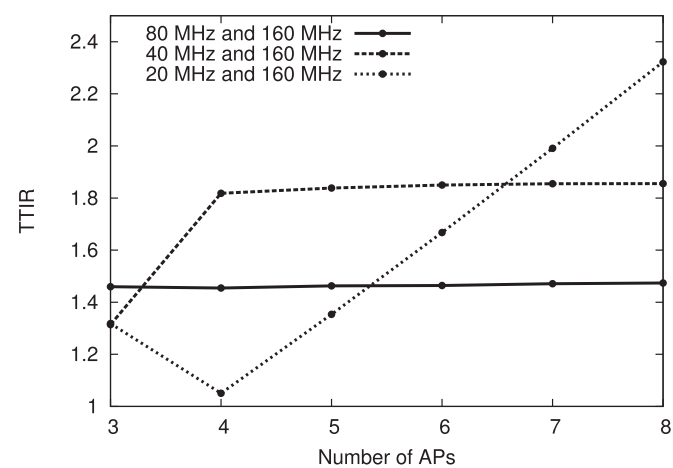

Fig. 14 Total throughput improvement ratio for all APs through coordination of APs 1 and 2.

1. It means that independent of the number of APs and the bandwidth of narrower channels, the total throughput of APs 1 and 2 is increased through the coordination of these APs in most cases. In Fig. 13, there are local minima whre the number of APs is four and the narrower bandwidth is $20 \mathrm{MHz}$, and the number of APs is six and the rarrower bandwidth is $40 \mathrm{MHz}$. This is because $\mathcal{E}\left(\widetilde{G}_{\mathrm{C}}\right)$ or $\mathcal{E}\left(\widetilde{G}_{\mathrm{I}}\right)$ is varied around these points.

To discuss the impact of coordination of APs 1 and 2 on the total throughput of all APs, we define the total TIR (TTIR) through coordination as the ratio between the worst equilibrium in $\widetilde{G}_{\mathrm{I}}$ and that in $\widetilde{G}_{\mathrm{C}}$ in terms of the total throughput as follows:

$$
\mathrm{TTIR}=\frac{\min _{\boldsymbol{a} \in \mathcal{E}\left(\widetilde{G}_{\mathrm{C}}\right)} \sum_{i \in\{1, \ldots, N\}} u_{i}(\boldsymbol{a})}{\min _{\boldsymbol{a} \in \mathcal{E}\left(\widetilde{G}_{\mathrm{I}}\right)} \sum_{i \in\{1, \ldots, N\}} u_{i}(\boldsymbol{a})}
$$

TTIR is shown in Fig. 14. The total throughput of all APs increases even if only two APs are coordinated. The reason of the total throughput improvement is the same as that discussed in Sect. 6.1.2., i.e., the coordination of APs eliminates the undesirable Nash equilibrium, where all APs selects the same $160 \mathrm{MHz}$ channel.

\section{Conclusion}

This paper analyzed the throughput of a multibandwidth channel selection by the coordinated APs for WLAN systems. The interactions of two coordinated APs and an uncontrollable AP were analyzed using game theory and the throughputs of Nash equilibria were evaluated. In particular, we formulated two situations as strategic-form games and compared them: (1) three APs independently select from multibandwidth channels to maximize their individual throughput and (2) a centralized controller coordinating two APs and an uncontrollable AP independently select from multibandwidth channels in which the centralized controller attempts to maximize the aggregated throughput of the two coordinated APs. One important result is that the two coordinated APs can increase their expected total throughput at 
Nash equilibria. In addition, we found that even if we could not control all of the APs, control of part of the APs has the potential to substantially decrease PoA. Additional evaluation with more than or equal to three APs also confirms throughput improvement through coordination of APs.

Although the strategic-form game is a so-called oneshot game and the simplest framework of game theory, we used the aforementioned conclusion for multibandwidth channel selection. Multibandwidth channel selection may also become an important technique in IEEE 802.11ah [14], where $1 \mathrm{MHz}, 2 \mathrm{MHz}, 4 \mathrm{MHz}, 8 \mathrm{MHz}$, and $16 \mathrm{MHz}$ channels will be supported. We emphasize that the purpose of this paper was to discuss the impact of the multibandwidth channel selection and coordination of APs rather than its practical algorithms. Thus, the detailed schemes for each AP to summarize the factors of games, i.e., the number of adjacent APs and utilities, as well as algorithms to achieve a Nash equilibrium are beyond the scope of this paper.

Future research is required to discuss more realistic assumptions as adjacent channel interference. Development of fair channel selection scheme is another future work because fairness among stations associated with the coordinated APs is not taken into account in the present paper.

\section{References}

[1] M. Park, "IEEE 802.11ac: Dynamic bandwidth channel access," Proc. IEEE International Conference on Communications (ICC'11), pp.1-5, OR, USA, June 2011.

[2] R.V. Nee, "Breaking the gigabit-per-second barrier with 802.11ac," IEEE Wireless Commun., vol.18, no.2, p.4, April 2011.

[3] IEEE Std 802.11n-2009, "Part 11: Wireless LAN medium access control (MAC) and physical layer (PHY) specifications: - Amendment 5: Enhancement for higher throughput," Oct. 2009.

[4] K.K. Leung and B.J.J. Kim, "Frequency assignment for IEEE 802.11 wireless networks," Proc. IEEE Vehicular Technology Conference (VTC'03), vol.3, pp.1422-1426, NJ, USA, Oct. 2003.

[5] R. Akl and A. Arepally, "Dynamic channel assignment in IEEE 802.11 networks," Proc. IEEE International Conference on Portable Information Devices (PORTABLE'07), pp.1-5, Denton, USA, May 2007.

[6] H. Luo, "A distributed dynamic channel allocation technique for throughput improvement in a dense WLAN environment," Proc. IEEE International Conference on Acoustics, Speech, and Signal Processing (ICASSP'04), vol.5, no.3, pp.345-348, NJ, USA, May 2004.

[7] Y. Lee, K. Kim, and Y. Choi, "Optimization of AP placement and channel assignment in wireless LANs," Proc. IEEE Local Computer Networks (LCN'02), pp.831-836, Seoul, South Korea, Nov. 2002.

[8] X. Ling and K. Yeung, "Joint access point placement and channel assignment for 802.11 wireless LANs," Proc. IEEE Wireless Communications and Networking Conference (WCNC'05), vol.3, pp.15831588, Chengdu, China, March 2005.

[9] A. Eisenblatter, H.F. Geerdes, and I. Siomina, "Integrated access point placement and channel assignment for wireless LANs in an indoor office environment," Proc. IEEE International Symposium on a World of Wireless, Mobile and Multimedia Networks (WoWMoM'07), pp.1-10, Helsinki, Finland, June 2007.

[10] Z. Han, D. Niyato, W. Saad, T. Basara, and A. Hjorungnes, Game Theory in Wireless and Communication Networks, Cambridge University Press, 2012.

[11] D. Fudenberg and J. Tioel, Game Theory, The MIT Press, 1991.

[12] A. Mas-Colell, M.D. Whinston, and J.R. Green, Microeconomic
Theory, Oxford Univ. Press, 1995.

[13] G. Bianchi, "Performance analysis of the IEEE 802.11 distributed coordination function," IEEE J. Sel. Areas Commun., vol.18, no.3, pp.535-547, March 2000.

[14] IEEE 802.11 Task Group ah (TGah) Sub 1 GHz license-exempt operation http://www.ieee802.org/11/Reports/tgah_updata.htm

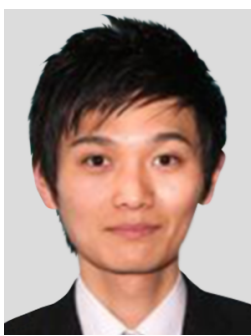

Kohei Hanada received the B.E. degree in electrical and electronic engineering from Kyoto University in 2011. He is currently studying toward his M.E. degree at the Graduate School of Informatics, Kyoto University. His research interests include game theory and wireless communication systems. He is a student member of the IEEE.

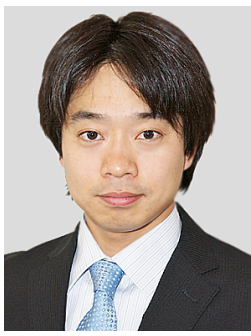

Koji Yamamoto received the B.E. degree in electrical and electronic engineering from Kyoto University in 2002, and the M.E. and Ph.D. degrees in informatics from Kyoto University in 2004 and 2005, respectively. From 2004 to 2005 , he was a research fellow of the Japan Society for the Promotion of Science (JSPS). Since 2005, he has been with the Graduate School of Informatics, Kyoto University, where he is currently an associate professor. From 2008 to 2009, he was a visiting researcher at Wireless@KTH, Royal Institute of Technology (KTH) in Sweden. His research interests include game theory, spectrum sharing, and M2M networks. He received the PIMRC 2004 Best Student Paper Award in 2004, the Ericsson Young Scientist Award in 2006, and the Young Researcher's Award from the IEICE of Japan in 2008. He is a member of the IEEE.

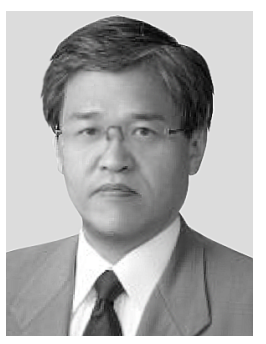

Masahiro Morikura received his B.E., M.E., and $\mathrm{Ph} . \mathrm{D}$. degrees in electronics engineering from Kyoto University, Kyoto, Japan in 1979, 1981 and 1991, respectively. He joined NTT in 1981, where he was engaged in the research and development of TDMA equipment for satellite communications. From 1988 to 1989, he was with the Communications Research Centre, Canada, as a guest scientist. From 1997 to 2002, he was active in the standardization of the IEEE 802.11a based wireless LAN. He received the Paper Award and the Achievement Award from IEICE in 2000 and 2006, respectively. He also received the Education, Culture, Sports, Science and Technology Minister Award in 2007 and Maejima Award in 2008. Dr. Morikura is now a professor in the Graduate School of Informatics, Kyoto University. He is a member of the IEEE. 


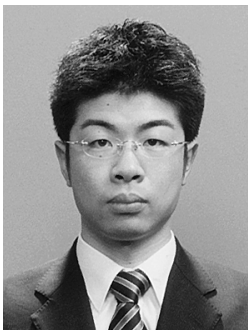

Koichi Ishihara received his B.E., M.E. and $\mathrm{Ph} . \mathrm{D}$. degrees in communications engineering from Tohoku University, Sendai, Japan, in 2004, 2006 and 2011, respectively. Since April 2006, he has been with NTT Network Innovation Laboratories, Yokosuka, Japan. His current research interests include digital signal transmission techniques for broadband wireless and fiber-optic communication systems. $\mathrm{He}$ received the Young Engineer Award from the IEICE in 2009, and the IEICE RCS (Radio Communication Systems) Active Research Award in 2011. He is a member of IEEE.

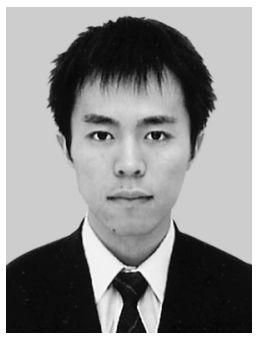

Riichi Kudo received the B.S. and M.S degree in geophysics from Tohoku University, Japan, in 2001 and 2003, respectively. In 2003, he joined NTT Network Innovation Laboratories, Yokosuka, Japan. He has been engaged in the MIMO communication systems and the beamforming methods. He received the Young Engineer Award from the Institute of Electronics, Information and Communication Engineers (IEICE), Technical Committee of Antenna and Propagation, Technical Committee on Optical Communication Systems, and IEEE AP-S Japan Chapter, Japan, in 2006, 2009, 2010, and 2011, respectively, and received the IEICE Best Paper Award in 2011. He is a visiting fellow at the Centre for Communications Research (CCR), University of Bristol. His current research interest is spatial signal processing techniques in massive MIMO systems. 